\title{
BIOCOMPATIBILITY OF CONVENTIONAL ACRYLIC RESIN VERSUS MODIFIED WITH TITANIUM DIOXIDE NANOPARTICLES IN MAXILLOFACIAL PROSTHESIS. A PARALLEL RANDOMIZED CONTROL TRIAL
}

\author{
Noha Ali Gamaleldin* and Nancy Nader El Sherbini*
}

\begin{abstract}
Statement of the problem: Biocompatibility of the maxillary obturators, is one of the most important bases for excellent prognosis. The modified acrylic resin with titanium dioxide nanoparticles has been shown to be an efficient form of poly (methyl methacrylate) resin for the fabrication of dentures.
\end{abstract}

Objective: The aim of this study is to evaluate the biocompatibility of the obturator constructed with the modified acrylic resin compared to the conventional one.

Materials and Methods: Fourteen patients with acquired maxillary defect were selected to participate in this study, the patients were divided randomly into two groups. Group I patients received obturators constructed from the conventional (poly methyl methacrylate) acrylic resin, Group II patients received obturators constructed from (poly methyl methacrylate) acrylic resin modified by the addition of titanium dioxide nanoparticles. Three swabs were taken from each patient (one from the fitting surface of the obturator facing the defect and the two others were obtained from the nasal and palatal surfaces of the defect) these swabs at 3 months and 6 months after insertion. The results were collected, tabulated and statistically analyzed.

Results: As regard the incidence of bacteria harbored in the defect there was an increase in the bacterial count throughout the follow up period in both groups. The increase was statistically significant in group 1 while was non-significant in group 2 .

Conclusion: The use of modified acrylic resin with titanium dioxide nanoparticles in obturators construction obviously improved its biocompatibility

KEY WORDS: Maxillary obturators, PMMA, Denture-base, Titanium nanoparticles, IgA

\footnotetext{
* Associate Professor Prosthodontic Department, Faculty of Dentistry, Cairo university, Cairo, Egypt
} 


\section{INTRODUCTION}

The biocompatibility of prosthetic materials of appliances used for the replacement or augmentation of human tissues has been a critical concern within the health care disciplines throughout the history of medicine and dentistry. ${ }^{(1)}$

The environmental conditions (oral, nasal secretions and bacterial flora) associated with prosthetic rehabilitation appliances of oral maxillofacial areas. These appliances are in intimate contact with a large area of oral mucosa that may be infected, inflamed or even lacerated. ${ }^{(2,3)}$

Several materials have been tried and were found to be suitable for clinical use and applications, such as hard or plasticized acrylic resin, latex rubber, polyurethane, silicon elastomers, chlorinated polyethylene and terpolymer acrylic latex ${ }^{(4-6)}$

Some investigators have listed the qualities and requirements of an ideal maxillofacial material; not physically affected by the soft tissue; not capable of initiating an inflammatory or foreign body reaction; be chemically inert, non carcinogenic. ${ }^{(7-9)}$.

By the evolution of the nanotechnology in the dental material, the mechanical properties of acrylic resins have been improved by chemical modification of existing PMMA with various nano fillers such as metallic powders, fibers, nanoparticles and nanorods. ${ }^{(10-12)}$

Several studies had investigated the effect of adding titanium dioxide nanoparticles on the properties of PMMA. It was concluded that it could improve the flexure strength, fracture toughness, hardness, thermal conductivity of PMMA and it reduces the water sorption. Adhesion between resin matrix and filler particles is very important in order to enhance the roughness and the composite properties. ${ }^{6-8}$

Many studies proved that the addition of nanoparticles to PMMA leads to increase hydrophobicity, reduce the adherence to biomolecules and improve antimicrobial properties of PMMA resins. Cell cultures for toxicity testing of dental materials were found to be a valuable tool for understanding their biological behavior while the change in the microorganisms count and level of immunoglobulin A in the secretion of subjects using the obturator were the indicator of the acquired immunity changes. ${ }^{(12-14)}$

The question raised in this study does the addition of titanium dioxide nanoparticles to the maxillofacial prosthesis will affect its biocompatibility?

\section{Aim of the Study}

Evaluation of the biocompatibility of modified acrylic resin with titanium dioxide nanoparticles compared with conventional acrylic resin in the construction of maxillofacial prosthesis.

\section{MATERIAL AND METHODS}

Based on data from a previous studies ${ }^{(14,15)}$, a power calculation was performed using $\mathrm{G}^{*}$ Power 3.1 software (Heinrich Heine University, Dusseldorf, Germany). The calculation indicated that the sample size for each group should be a minimum of 10 cases. Thirty patients were selected from the outpatient maxillofacial clinic, Prosthodontic Department, Faculty of Dentistry.

\section{Inclusion criteria:}

- They had acquired hemi-maxillectomy defects due to surgical removal of tumors.

- The wound should be completely healed.

- Non smokers

- Participants of both sexes requiring dentures

\section{Exclusion criteria:}

The patients were not under antibiotics therapy, chemotherapy nor radiotherapy.

Randomization: After selection of the patients following the inclusion and exclusion criteria the patients were recruited by simple randomization 
in a 1:1 ratio into two groups using Randomizer software, Group I received maxillary obturator constructed from the conventional acrylic resin (polymethyl-methacrylate) (DPI Bombay Burmah Trading Corporation Ltd.), while group II received obturators constructed from the modified acrylic resin (polymethyl-methacrylate with the titanium dioxide nanoparticles AEROXIDE® TiO2 P25 (5\% by weight). The participants were unaware of the type of denture received.

This research was approved according to Declaration of Helsinki, signed consent forms were obtained from all participants before conducting the study and the Consolidated Standards of Reporting Trials (CONSORT) guidelines were followed.

The patients were patch tested with the two types of the acrylic resin to exclude the possibility of allergy.

Obturators were constructed following conventional manner, till the packing steps. For the first group the powder of the acrylic resin and the monomer were mixed according to the manufacturer's instructions, when it reached the dough stage it was packed on the master cast. While for the second group $5 \%$ by weight titanium dioxide nanoparticles were mixed thoroughly to the acrylic powder, then they were mixed with the monomer till it reach the dough stage and it was packed on the master cast.

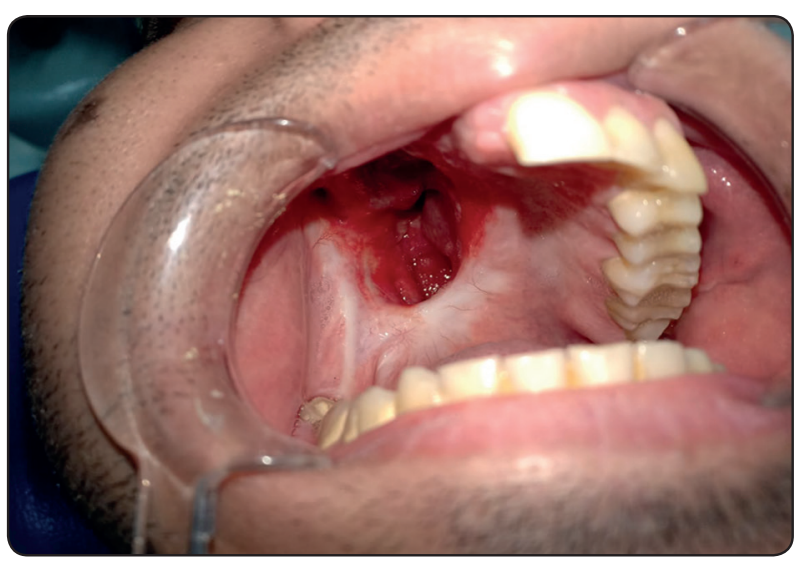

Fig. (1) Completely healed surgical cavity
Processing was done by direct immersion of the packed flasks in boiling water immediately after packing for a short time then the water was brought back to a full boil for 20 minutes.

Processed obturators were removed from the flasks and laboratory remount was done for both types to correct the occlusal errors that have occurred during processing.

All patients of both groups were given a sheet of oral hygiene instructions and were trained on insertion and removal of the obturators and how they could keep their obturator and the defect area clean. (Fig 1\&2)

\section{Bacteriological Procedures:}

At the time of insertion of the obturators, two swabs were taken from patients of each group, one swab from the nasal surface and another from the palatal surface of the defect. The two samples were considered as a base line (control samples) for patients in each group.

AFTER insertion three swabs were taken, one from the fitting obturator surface, nasal and palatal surface of the defect. These swabs were taken after 3 months and after 6 months for follow- up.

Each swab was immediately inoculated into a tube containing $1 \mathrm{ml}$ saline and added to $9 \mathrm{ml} \mathrm{sa}$ line after shaking to make a dilution of $1: 10$.

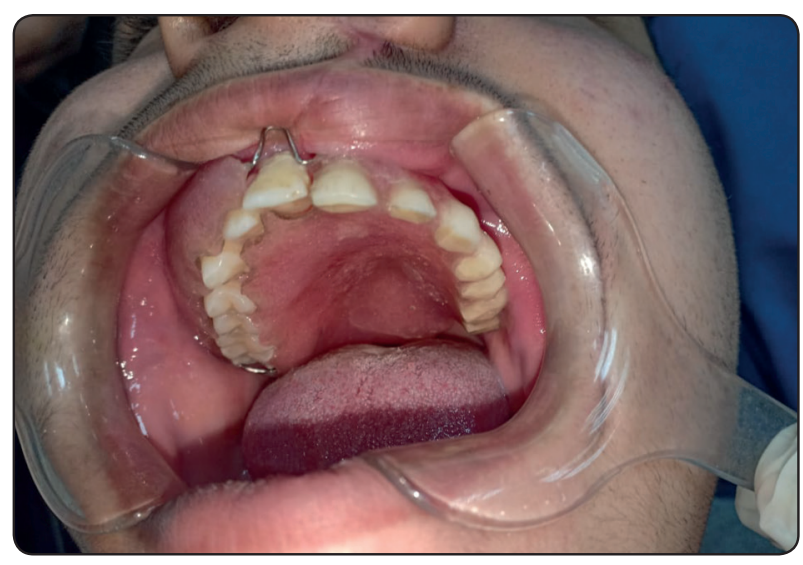

Fig. (2) Obturator constructed with modified acrylic resin 
The previous step was repeated to reach a dilution of $1: 1000$,then $0.1 \mathrm{ml}$ was transferred from the last dilution(1:1000) and plated onto two non- selective blood agar medium plates, one plate was incubated aerobically for 24 hours in an air incubator, while the other plate was anaerobically incubated for three days at $37^{\circ} \mathrm{C}$ using the gas pack anaerobic system. Total viable bacterial count (in $\mathrm{ml}$ ) was calculated by multiplying.

The number of colonies was $10 \times 10^{3}$. The colonies on aerobic plates were identified by their morphology, type of hemolysis, microscopic appearance and biochemical reactions. Colonies which appeared on anaerobic plates only were confirmed to be pure isolates of obligate anaerobes to be further identified by biochemical tests which included susceptibility to special potency antibiotic discs, nitrate reduction test, as well as growth in $20 \%$ bile.

\section{Detection of IgA in the defect secretions:}

Oral secretion samples from the defect were collected through a special capillary graduated tube, centrifuged at 1.550 R.P.M The supernatant fluid was then transformed into another sterile tube ready for investigation.
Detection of IgA level was done by single radial immunodiffusion using Behing-werke measuring viewer.12 wells were supplied in each plate to receive the examined samples which were allowed to diffuse in the wells of the plate under the effect of an electric current $(2 / 3 \mathrm{v} / \mathrm{cm})$ for 16 hours. The plates were stained with Coomassie brilliant blue for 5 minutes and washed with ethanol acetic acid solution till clear black ground appear.

Two way ANOVA test was used and the TukeyKramer Multiple Comparisons test were used to investigate significant differences among the groups.

\section{RESULTS}

These results were obtained from patients in both groups who had an obturator processed by conventional PMMA for group I and by modified acrylic resin with titanium dioxide nanoparticles for group II. The prevalence of bacteria and fungi cultured from the defect secretions of all cases and the order of their frequency are listed in the tables $(1,2,3 \& 4)$ illustrated in histograms (Fig:3,4).

TABLE (1): Number of colonies of aerobic organisms (CFU/ml)isolated from swab samples of both types of obturators during the follow up period.

\begin{tabular}{lcccccc}
\hline \multirow{2}{*}{ ORGANISM } & \multicolumn{2}{c}{ At insertion } & \multicolumn{2}{c}{3 months } & \multicolumn{2}{c}{6 months } \\
\cline { 2 - 7 } & Conventional & Modified & Conventional & Modified & conventional & modified \\
\hline Staphylococci species & 120 & 118 & 135 & 129 & 180 & 142 \\
Streptococci & 86 & 82 & 97 & 90 & 120 & 98 \\
Anthorocoid & 25 & 17 & 28 & 18 & 38 & 20 \\
Neisseria & 70 & 48 & 79 & 52 & 88 & 57 \\
Pneumonia & 12 & 12 & 12 & 13 & 16 & 14 \\
Gram-ve bacilli & 12 & 13 & 13 & 14 & 18 & 15 \\
Candida & 20 & 20 & 22 & 22 & 30 & 24 \\
\hline
\end{tabular}


As regard the incidence of bacteria harbored in the cavity it is obvious that there was increase in the total bacterial count throughout the follow up period in both groups. The increase was statistically significant in group I while it was nonsignificant in group II.

Regarding group I (conventional) there was statistically significant increase in the total bacterial count throughout the follow up period (at insertion, 3 months \& 6months) $p$ value $<0.001$.

Regarding group II (modified) there was an increase in the total bacterial count through out the whole follow up period with $\mathrm{p}$ value $<0.01$.

\section{When comparing both groups:}

At insertion; there was no significant difference in the total bacterial count between both groups, $\mathrm{p}$ value was 0.061 .

At 3 months; group II showed significantly lower total bacterial count compared to group II the $\mathrm{p}$ value was 0.01 .

At 6 months group II showed significantly lower total bacterial count compared to group II the $\mathrm{p}$ value was 0.001 .(Table 3 )

Regarding the amount of $\operatorname{IgA}$ in the two groups throughout the 6 months follow up period, table 4 shows insignificant difference between the two groups at insertion, while after 3 months and 6 months the difference was significant $\mathrm{p} \leq 0.01$.

TABLE (2): Number of colonies anaerobic organisms (CFU/ml) isolated from swab samples of both types obturators during the follow up period

\begin{tabular}{lcccccc}
\hline \multirow{2}{*}{ ORGANISM } & \multicolumn{2}{c}{ At insertion } & \multicolumn{2}{c}{3 months } & \multicolumn{2}{c}{6 months } \\
\cline { 2 - 7 } & Conventional & Modified & Conventional & Modified & Conventional & Modified \\
\hline Pigmented bacteroids & 37 & 35 & 42 & 38 & 56 & 42 \\
Fusiform bacilli & 25 & 17 & 28 & 27 & 18 & 20 \\
Borrelia & 12 & 13 & 14 & 13 & 14 & 15 \\
Spore forming group (+ve ) bacilli & 78 & 61 & 88 & 67 & 118 & 75 \\
\hline
\end{tabular}

TABLE (3): Showing the changes in the bacterial count $(\mathrm{CFU} / \mathrm{ml})$ in both groups through out the follow up period.

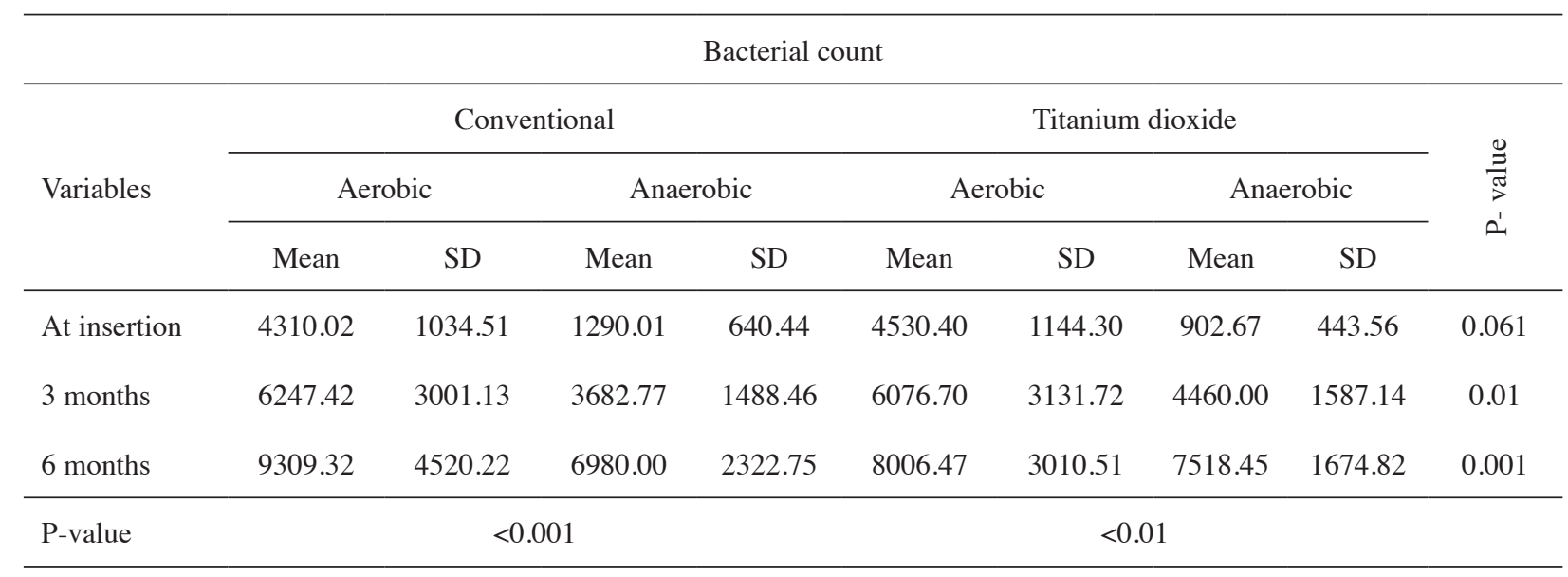


TABLE (4): Statistical analysis of IgA amount $(\mathrm{mg} / \mathrm{dl})$ in both group during the follow up period

\begin{tabular}{lccccc}
\hline \multirow{2}{*}{ ORGANISM } & \multicolumn{2}{c}{ Conventional } & \multicolumn{2}{c}{ Modified } & P-value \\
\cline { 2 - 5 } & Mean & SD & Mean & SD & 0.12 \\
\hline At insertion & 160 & 15 & 150 & 13 & 0.05 \\
3 months & 282 & 21 & 218 & 21 & 0.01 \\
6 months & 450 & 20 & 322 & 21 & \\
\hline
\end{tabular}

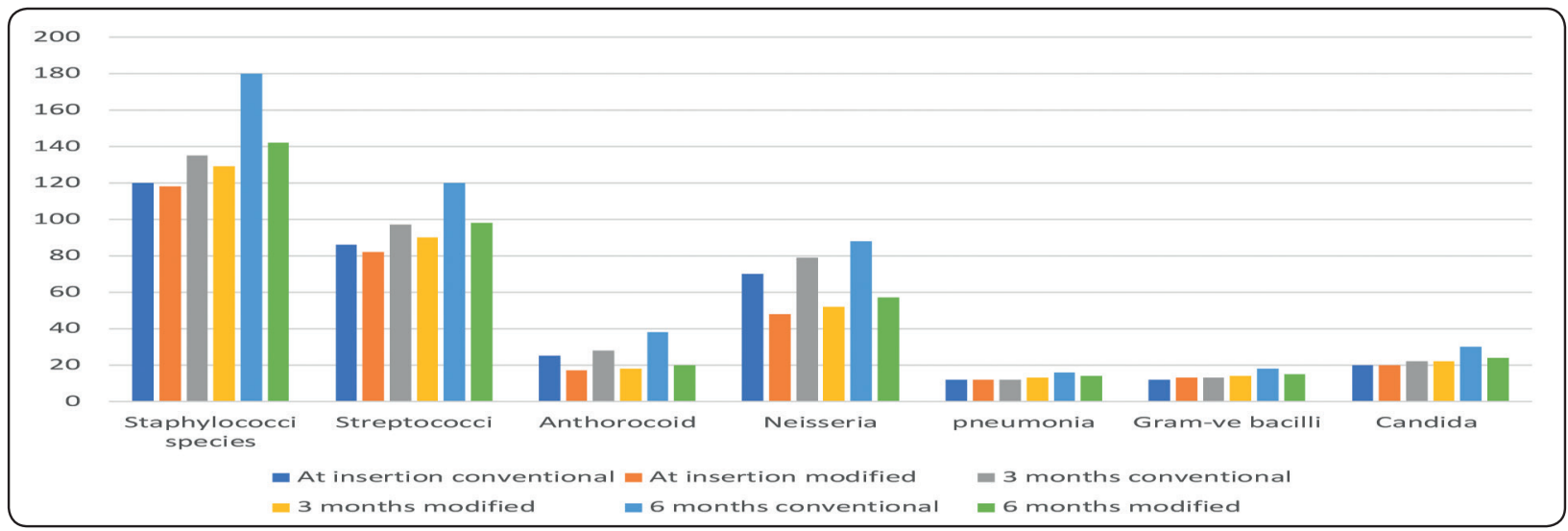

Fig. (3): Histogram showing the aerobic organisms isolated from swab samples of both types of obturators during the follow up period.

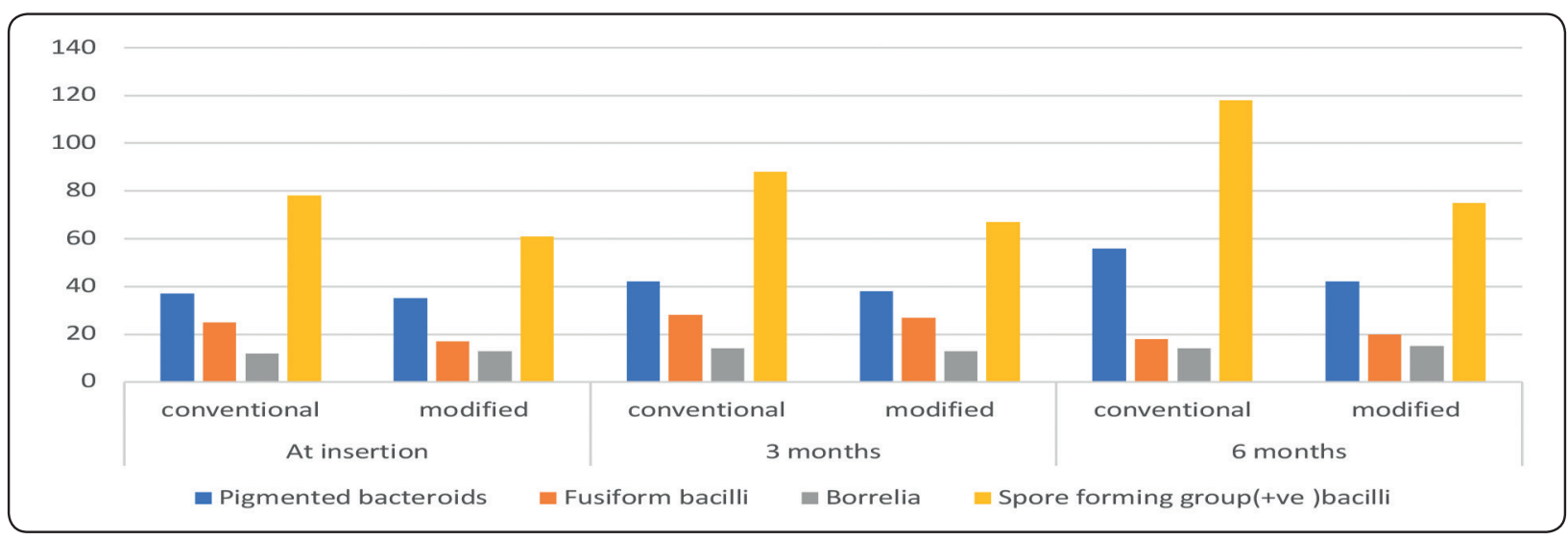

Fig. (4) : Histogram showing the anaerobic organisms isolated from swab samples of both types of obturators during the follow up period. 


\section{DISCUSSION}

Maxillofacial defects are considered very critical regarding both functional and biological qualities due to the challenging circumstances associated with such defects (pattern of movements and the nature of the surrounding environment). Insertion of any prosthesis is associated with change in the microbial flora. The degree of change varies according to the material, type, and design of the prosthesis. It was proved that presence of prosthesis within the oral cavity may alter the nature of the microbial flora due to difficulty in cleansing especially in deep inaccessible areas as in an obturator. ${ }^{10,11}$ Many attempts were carried out to overcome acrylic resin drawbacks and to improve its biological properties and tissue tolerance resulting in recently introduced denture base materials or modification in its composition and processing techniques. In this study, bacteriological and immunological investigations were used to evaluate the biocompatibility of modified acrylic resin with titanium dioxide nanoparticles used in the construction of an obturator. The results of this study showed an increase in the total microbial count (aerobic and anaerobic) after the usage of the obturators in both study groups, but with different degrees. Obturators fabricated from conventional heat cured resin modified by titanium dioxide nanoparticles showed reduction in the number of aerobic and anaerobic bacteria than those fabricated from conventional heat cured resin. ${ }^{10}$

Recent Studies confirmed aerobic and anaerobic microbial growth on the dentures and showed that different prostheses did not have the same spectra of microorganisms..$^{(\mathbf{1 0}, 11)}$

Staph-aureus was the main dominant kind of aerobic bacteria this was in agreement with the results of the previous studies, which could be attributed to the saliva alteration and the environments under the obturators. The most dominant anaerobic bacteria in all samples were the black pigmented Bacteroides and gram +ve spore forming bacilli. ${ }^{(12,13)}$
At the end of the follow up period the percentage of most organism was found to be greater in the samples obtained from patients of group I than those of group II and the difference was statistically significant. This could be attributed to decreased porosity associated with the addition of the titanium dioxide nanoparticles in the acrylic resin powder. ${ }^{(14-16)}$ A high percentage of anaerobic growth distribution was detected in group I than in group II this can attributed to the homogeneity and less porosity of group II obturators. These results are in agreement with several studies which concluded that the nanoparticles eliminate external and internal porosity resulting in a reasonable density of the acrylic resin. ${ }^{(17-19)}$ In addition the titanium oxide nanoparticles had an antibacterial action which explains the difference in the lower affinity of the material to harbour microorganisms. ${ }^{20}$ After the 6 months of the follow up period the amount of IgA of the group II was less than that of group I, which could indicate better tissue tolerance and acceptance of the obturator by the surrounding tissues. Mucous and nasal secretions may stagnate in the space between the fitting surface of obturator and the cavity, this may explain the increase of $\operatorname{IgA}$ in group I than group II which is more adaptable. ${ }^{(21,22)}$

Gradual increase of IgA in group I may be also due to local irritation causing delayed hypersensitivity from the released residual monomer that may produce a degree of cytotoxicity. This may explain the relationship between the presence of staphylococcus aureus and increasing the $\operatorname{IgA}$, as the relevance to protections of bacteria depends on the mechanism of pathogenicity. ${ }^{(23,24)}$

\section{CONCLUSION}

Within the limitations of this study, it was concluded that:

- The use of modified acrylic resin with titanium dioxide nanoparticles in obturators construction showed better biocompatibilty regarding the total bacterial count compared to the conventional PMMA alone. 
- The use of modified acrylic resin with titanium dioxide nanoparticles in obturators construction showed better tissue reaction regarding the total IgA compared to the conventional PMMA alone.

\section{CONFLICT OF INTEREST}

No potential conflict of interest relevant to this article was reported.

\section{FUNDING}

This research did not receive any specific grant from funding agencies in the public, commercial, or not-for-profit sectors.

\section{REFERENCES}

1. Vieira CLZ, Caramelli B. The history of dentistry and medicine relationship could the mouth finally return to the body? Oral Disease J 2009;15:538-546

2. Jeroen P J, Dings Matthias AW, Merkx. Maxillofacial prosthetic rehabilitation: A survey on the quality of life. $\mathrm{J}$ Prosthet Dent 2018; 120:780-786

3. Mahajan H, Gupta K Maxillofacial Prosthetic Materials: A Literature Review J. Orofac Res.2012;2:87-90

4. Ashour M, ElShennawy M, Althomali Y, Omar AA. Effect of Titanium Dioxide Nano Particles incorporation on Mechnical Physical Properties of Two Different Types of Acry;ic resin Denture Base. World J Nano Sci Eng .2016; 6:111-119

5. Altarawneh S, Bencharit S, Mendoza L, Curran A, Barrow D, Barros S, Preisser J, Loewy ZG, Gendreau L, Offenbacher S. Clinical and Histological Findings of Denture Stomatitis as Related to Intraoral Colonization Patterns of C. albicans, Salivary Flow, and Dry Mouth. J Prosthodont. 2013;22: 13-22.

6. Dias HB, Bernardi MIB, Bauab TM, Hernandes AC, A.N. Souza Rastelli AN.Titanium dioxide and modified titanium dioxide by silver nanoparticles as an anti biofilm filler content for composite resins. Dent Mater 2019;35:e36-e46

7. Daniluk T., Fiedoruk K., Sciepuk M. Aerobic bacteria in the oral cavity of patients with removable dentures. Advances in Medical Sciences. 2006; 51: 86-91.
8. Raustia A. PMMA denture base material enhancement: a review of fiber filler and nanofiller addition. Int $\mathrm{J}$ Nano medecine. 2017;12:3801-3812

9. Anehosur GV, Kulkarni RD, Naik MG, Nadiger RK. Synthesis and determination of antimicrobial activity of visible light activated $\mathrm{TiO} 2$ nanoparticles with polymethyl methacrylate denture base resin against Staphylococcus aureus. J Gerontol Geriatric Res. 2012;1:2-8.

10. Koch C., Burgers R., Hahnel S. Candida albicans adherence and proliferation on the surface of denture base materials. Gerodontology. 2013; 30:309-313.

11. Chenc, Ren W, Gaol, ChengZhuang L. Function of obturator prostheses after maxillectomy and prosthetic obturator rehabilitation. Braz J. OTO rhinolarygo. 2016; 82:177-183

12. Olms C, Yahiaoui-Doktor M, Remmerbach TW, Stingu CS. Bacterial Colonization and Tissue Compatibility of Denture Base Resins. Dent J (Basel). 2018;15:20-32.

13. Deepthi PV, Fernandez T, Karthikeyan S. Oral microbilogical flora in denture wearers -a review. J Adv Med Dent Sci Res. 2016; 4:92-94.

14. McCord Contemporary technique for denture base fabrication. J Prosthodont. 2009;18:106-111

15. Sriram N, Jain AR. Assessment of salivary PH and microbial growth in patients wearing complete denture. Drug Invention Today. 2018;10:488-419.

16. Kul E, Aladag LI and Yesildal. Evaluation of thermal conductivity and flexural strength properties of poly (methyl methacrylate denture) base material reinforced with different fillers. Prothet. Dent J, 2016;116:803-810.

17. Kakoei S, Hosseini B, Haghdoost A, Sanjari M, Gholamhosseinian A., Vahid F N. Evaluation of salivary secretory immunoglobulin A levels in diabetic patients and association with oral and dental manifestations. SQU Medical J. 2015;15:e507-e511.

18. Biswas S, Rolain JM. Use of MALDI-TOF mass spectrometry for identification of bacteria that are difficult to culture. J Microbiol Meth. 2013; 92:14-24.

19. Arathi K, Prasad G, Shetty SJ, Iype AK. Uncultivable Bacteria in the Oral Cavity: A Review. Int J Adv Health sci. 2015; 1:16-19.

20. Sodagar A, Bahador A, Khalil S, Shahroudi AS, Kassaee MZ The effect of $\mathrm{TiO}_{2}$ and $\mathrm{SiO}_{2}$ nanoparticles on flexural strength of poly(methyl methacrylate) acrylic resins. J Prosthodont Res. 2013; 57:15-19. 
21. Esteban FFI, Hiers RD, P. Larso JohnsonM, E. O'Rear, RondinoneAJ Antibacterial dental adhesive resins containing nitrogen-doped titanium dioxide nanoparticles. Mater Sci Eng C Mater Biol Appl.2018;93 :931-943

22. Kul E, Aladag L., I and Yesildal R. Evaluation of thermal conductivity and flexural strength properties of poly (methyl methacrylate denture) base material reinforced with different fillers J Prosthet. Dent.2016;116:803-810
23. Gomaa AM, Lamfon HA and yahia a. Maher YA. Microbiological and immunological effects of monomer free thermosens and nano zirconia oxide reinforced denture base resins on controlled diabetic denture wearers. Egy Dent J. 2019; 65, 563:577

24. Rashid H, Sheikh Z, Vohra F. Allergic effects of the residual monomer used in denture base acrylic resins. Eur. J Dent. 2015; 9:614-619. 